\section{NUEVAS ÁREAS DE DISTRIBUCIÓN DE Aedes aegypti EN PERÚ}

\author{
NEW DISTRIBUTION AREAS OF Aedes aegypti \\ IN PERU
}

\author{
Edwin Requena-Zúñiga1,a, Leonardo Mendoza-Uribe ${ }^{1, a}$, \\ Mónica Guevara-Saravia ${ }^{2, a}$
}

Sr. Editor. En el Perú, Ae. aegypti se encuentra distribuido principalmente en la región selvática y en la costa norte, llegando hasta la región Lima ${ }^{(1)}$, no se conoce ciudades o localidades al sur de Lima que puedan estar infestadas con este vector. La influencia del clima y las variables medioambientales como las bajas temperaturas y poca precipitación pluvial son consideradas como factores que retardarían la introducción y posterior establecimiento de Ae. aegypti en algunas regiones, como es el caso de la sierra y la costa sur del país, la altitud también puede considerarse como una limitante para la distribución de este vector, pocos estudios reportan la presencia de Ae. aegypti en localidades mayores a $1700 \mathrm{~m}$ de altitud.

Las hembras de Ae. aegypti son antropofílicas, están adaptadas al entorno urbano, descansan en las zonas internas y oscuras de las casas ${ }^{(2)}$, y tienen la facilidad de oviponer en una gran diversidad de contenedores. Estas adaptaciones a los ambientes humanos junto con la longevidad y la resistencia de sus huevos a la desecación ayudan a la infestación de este vector a nuevas áreas, transportada pasivamente por acción del hombre ${ }^{(3)}$. Cuando se realiza presión mediante el uso de químicos para el control vectorial de Ae. aegypti en ciudades grandes y pobladas, es probable que este vector se disperse a localidades nuevas, ayudadas por las vías de conexión, también es probable que antes de infestar localidades pequeñas primero se establezca en ciudades con condiciones favorables para su proliferación, como alta concentración de personas, gran intercambio comercial, cercanía a carreteras principales, puertos y otros ${ }^{(4)}$.

El hallazgo de Ae. aegypti en nuevas regiones del Perú en los últimos años, posiblemente no se deba a una introducción reciente del vector, sino a una progresiva expansión hacia áreas favorables para su desarrollo. La vigilancia entomológica poco oportuna puede contribuir a una demora en el hallazgo del Ae. aegypti en nuevas localidades, con el consecuente riesgo de que una localidad o región en la cual no se conocía su presencia, pueda ocurrir la aparición de casos de dengue, tal como ha sucedió en el distrito de Ayna, Ayacucho en el $2015^{(5)}$.

Durante la tercera semana de mayo de 2015, en la Localidad de Chulqui, (0949'58.57'S, 7606'00.55'W) perteneciente al distrito de Churubamba, ubicada aproximadamente a $23 \mathrm{~km}$ de la ciudad de Huánuco, se colectaron larvas y pupas de culicineos sospechosos de ser Ae. aegypti los que se encontraron en contenedores abandonados (llantas con agua). Posteriormente, se realizó una búsqueda complementaria en la misma ciudad de Huánuco $\left(09^{\circ} 55^{\prime} 36.9^{\prime \prime}\right.$ S, 76¹3'59.6" O) y en los almacenes de vehículos usados de la Municipalidad Provincial ubicados en la localidad de Pillco Marca (0958'26.7" S, 76¹4'41.0" O), en donde se colectaron un total de 421 larvas y 123 pupas.

En la región Ica, la búsqueda de $A$ e. aegypti se realizó por la sospecha de un probable caso de dengue autóctono en el distrito de Parcona, las colectas de las muestras (posibles de ser Ae. Aegypti) fueron realizados entre el 30 de mayo y 3 de junio de 2015, las inspecciones larvarias se realizaron en las viviendas del distrito de Parcona (14 ${ }^{\circ} 02^{\prime} 41.96$ " S, $75^{\circ} 42^{\prime} 05.89^{\prime \prime}$ O) ubicado en la Ciudad de Ica, en donde se colectaron 109 larvas y 2 pupas, cuyos criaderos fueron bidones, cilindros, pozas y floreros.

Todas las muestras fueron transportadas al Laboratorio de Entomología del Instituto Nacional de Salud, en la ciudad de Lima, en donde se realizó la identificación taxonómica, confirmando la presencia de Ae. aegypti en la región Ica y la ciudad de Huánuco. Con este reporte, la región lca se constituye en la región número 20 del Perú en estar infestada con este vector.

Una probable vía de dispersión hacia la región Ica puede haber sido a través de Lima ${ }^{(6)}$, pero también a través de la carretera Libertadores Wari desde el valle de los ríos Apurímac, Ene y Mantaro (VRAEM), por lo que las ciudades que están a lo largo de esta vía también pueden estar infestadas. Asimismo, la presencia de $A e$. aegypti a $1900 \mathrm{~m}$ de altitud en la ciudad de Huánuco, representa la de mayor altitud reportada en el Perú.

\footnotetext{
Laboratorio de Entomología, Instituto Nacional de Salud. Lima, Perú

Dirección Ejecutiva de Salud Ambiental, Dirección Regional de Salud de Ica. Ica, Perú

Biólogo

Recibido: 29-12-15 Aprobado: 09-03-16
}

Citar como: Requena-Zúñiga E, Mendoza-Uribe L, Guevara-Saravia M. Nuevas áreas de distribución de Aedes aegypti en Perú [carta]. Rev Peru Med Exp Salud Publica. 2016;33(1):171-2. doi: 10.17843/rpmesp.2016.331.1804 
Se recomienda realizar estudios sobre la dispersión del Ae. aegypti en zonas costeras del sur del Perú, así como en regiones de altura donde aún no se ha detectado su presencia, a fin de tomar las medidas de prevención y control adecuadas en forma oportuna.

Agradecimientos: a Edgard Reina por su apoyo en facilitar las instalaciones de la Dirección Ejecutiva de Salud Ambiental de Tingo María y equipos de identificación. A los técnicos de laboratorio Ruth Quiroz Morales y Anthony Palomino, por su colaboración en la colecta de las larvas en la ciudad de Huánuco. A María Ofelia Fernández Rodríguez por brindarnos facilidades en la utilización del ambiente y uso de equipos de identificación de la Dirección Ejecutiva de Salud Ambiental de Ica. A los inspectores sanitarios de la Dirección Ejecutiva de Salud Ambiental de Ica. A Fernando Donaires por sus sugerencias en la redacción de la presente carta.

Fuentes de financiamiento: autofinanciada.

Conflictos de interés: los autores declaran no tener conflictos de interés.

\section{REFERENCIAS BIBLIOGRAFICAS}

1. Andrade CS, Cáceres AG, Vaquerizo A, Ibáñez-Bernal S, Cachay LS. Reappearance of Aedes aegypti (Diptera: Culicidae) in Lima, Peru. Mem Inst Oswaldo Cruz. 2001;96(5):657-8. doi: 10.1590/S0074-02762001000500011
2. Espinoza F. Dinámica de transmisión del dengue en la ciudad de Colima, México [tesis para doctorado]. México: Facultad de Ciencias Biológicas y Agropecuarias. Universidad de Colima; 2002.

3. Wong J, Stoddard ST, Astete H, Morrison AC, Scott TW. Oviposition site selection by the dengue vector Aedes aegypti and its implications for dengue control. PLoS Negl Trop Dis. 2011;5(4):e1015. doi: 10.1371/journal.pntd.0001015

4. Guagliardo SA, Barboza JL, Morrison AC, Astete H, VazquezProkopec C, Kitron U. Patterns of geographic expansion of Aedes aegypti in the Peruvian Amazon. PLoS Negl Trop. 2014;8(8):e3033. doi: 10.1371/journal.pntd.0003033

5. Perú, Ministerio de Salud, Dirección General de Epidemiología. Vigilancia en medios de comunicación para detectar brotes, epizootias, desastres y otras emergencias sanitarias [Internet]. Reporte $\mathrm{N}^{\circ}$ 090-2015. Lima, Minsa; 2015 [citado el 15 de setiembre de 2015]. Disponible en: http://www.dge.gob.pe/ portal/docs/rumores/2015/Reporte\%20090-2015.pdf

6. Cabezas C, Fiestas V, García-Mendoza M, Palomino M, Mamani E, Donaires F. Dengue en el Perú: a un cuarto de siglo de su reemergencia. Rev Peru Med Exp Salud Pública. 2015;32(1):146-56. doi: 10.17843/rpmesp.2015.321.1587
Correspondencia: Edwin Requena Zuñiga

Dirección: Av. Defensores de morro \# 2280.

Teléfono: (511) 986697350

Correoelectrónico:erequena@ins.gob.pe

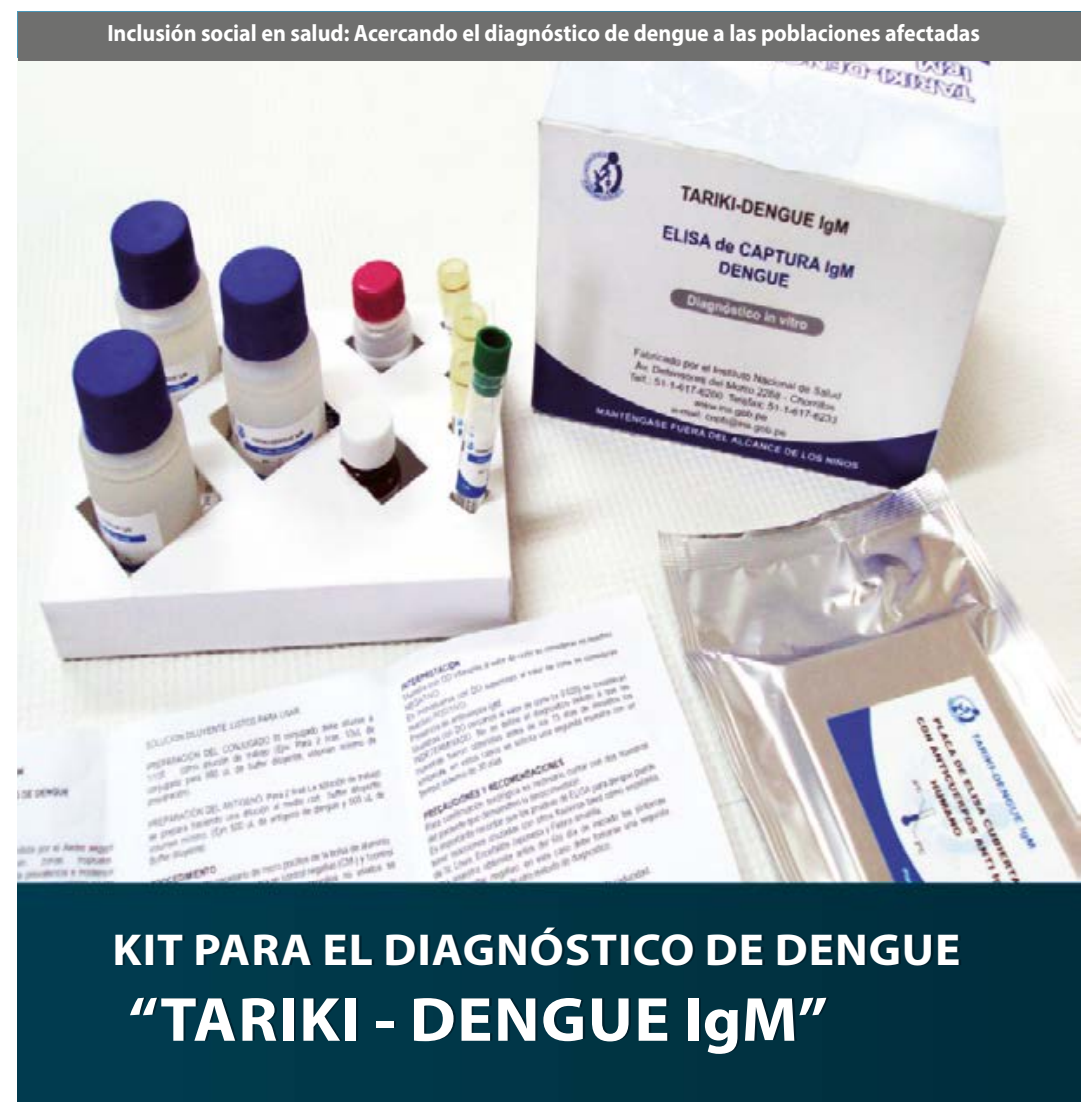

